\title{
Inhibition of Colon Cancer Cells Proliferation and Invasion by Etoposide Loaded on Nano-Suspensions Containing Black Currant Seed and Evening Primrose Oils
}

\author{
SALWA M AL-HASHEMI, MAYSON H ALKHATIB* AND HANA MOHAMED AHMMED GASHLAN \\ Department of Biochemistry, Faculty of Science, King Abdulaziz University, Jeddah, Saudi Arabia
}

Hashemi et al.: Inhibition of Colon Cancer Cells Proliferation and Invasion

\begin{abstract}
Formulating etoposide into a nanoemulsion based on combining two natural oils, Ribes nigrum (black currant) seed oil and organic evening primrose oils may improve its antineoplastic activity in cancer cells. The present study aimed to synthesize, characterize and in vitro evaluate the cytotoxic effect of formulating etoposide-loaded (black currant) seed oil and organic evening primrose oils on Colorectal Carcinoma colon cancer cells. The anti-proliferation and anti-invasion effects of the produced formula on Colorectal Carcinoma were investigated by the cell counting kit-8 and the collagen-based cell invasion assay, respectively. Mitochondrial staining kit, annexin Fluorescein isothiocyanate double staining, and nucleosome detection were used to discriminate the stages of apoptosis. According to the dynamic light scattering results, formulating etoposide-loaded (black currant) seed oil consisted of nanodroplets with z-average diameters of $144.6 \pm 5.3 \mathrm{~nm}$ and mean surface charge of $-\mathbf{0 . 0 8 1} \pm 0.001 \mathrm{mV}$. Only $(1.7 \pm 1.3)$ $\mu \mathrm{M}$ of formulating etoposide-loaded (black currant) seed oil was able to cause $50 \%$ reduction in the Colorectal Carcinoma cellular growth which was strikingly lower than that of free- formulating etoposide $(18.1 \pm 2.5 \mu \mathrm{M})$ by around ten-fold. The superior apoptotic effect of Formulating etoposide Ribes nigrum (black currant) seed oil and organic evening primrose oils relative to free- formulating etoposide on the Colorectal Carcinoma cells was ascertained by the enhanced mitochondrial depolarization, higher nucleosomal ratio and increased amount of early and late apoptotic cellular populations. The Formulating etoposide Ribes nigrum (black currant) seed oil and organic evening primrose oils treatment impeded the invasion of colorectal carcinoma cells by $(54.8 \pm 1.2) \%$, which was greater than the free- formulating etoposide treatment that deterred only $(35.1 \pm 1.2) \%$ of the invaded cells. In conclusion, the incorporation of formulating etoposide into a nanoemulsion formulated from the bioactive natural oils potentiates its antitumor activity in colon cancer cells.
\end{abstract}

Key words: Natural oils, polyunsaturated fatty acids, nanocarrier, zetasizer measurement, nucleosomes, apoptosis

According to the statistical report on cancer introduced by GLOBOCAN 2018, colon cancer represents the most common malignant tumors diagnosed worldwide. It ranks the third in terms of incidence while in terms of mortality, colon cancer arises to the second common causes leading to the death following lung cancer ${ }^{[1-3]}$. In addition to the drug resistance, metastasis is the main obstacle for effective therapies, which could lead to increasing the death rate of colon cancer patients ${ }^{[4-5]}$.

Chemotherapy is one of the possible options available for cancer treatments. In general, continuous exposures to the chemotherapeutic agents for a prolonged period

*Address for correspondence

E-mail: mhalkhatib@kau.edu.sa

May-June 2021 are obliged for effective cancer therapy, which is not feasible for formulating etoposide (ETP) owing to the lower solubility, short biological half-life and bioavailability ${ }^{[6]}$. Moreover targeting and efficient delivery of the anti-cancer agent at the tumor site, while preventing severe damage is the main hurdle in cancer

This is an open access article distributed under the terms of the Creative Commons Attribution-NonCommercial-ShareAlike 3.0 License, which allows others to remix, tweak, and build upon the work non-commercially, as long as the author is credited and the new creations are licensed under the identical terms

Accepted 30 May 2021

Revised 06 February 2021

Received 09 January 2020

Indian J Pharm Sci 2021;83(3):465-472 
diagnosis and therapy ${ }^{[7]}$. Therefore, it is necessary to propose new deliveries for the chemotherapeutic agents that improve their selectivity at the target site.

Polyunsaturated fatty acids (PUFAs) are one of the most natural oils that possess anti-cancer and antimicrobial activity. The beneficial properties of PUFAs could be ascribed to their safety, high efficacy, a broad range of activity and absence of resistance mechanisms. Furthermore, it could be used as a tumor-specific ligand to guide antineoplastic agents selectively at the tumor site without affecting the other healthy cells ${ }^{[8-10]}$.

Nanoemulsion with the mean of the small droplet size ranging from $20-200 \mathrm{~nm}$ is one of the most promising nano-delivery systems. Nanoemulsions are dispersion systems that consist of water and oils, stabilized by emulsifying agents, surfactants and cosurfactants. It has numerous advantages as nanocarrier, which include targeting drug delivery, stability, enhancing the solubility of the hydrophobic drug and providing protection of drugs from degradation ${ }^{[11-12]}$. Although ETP was loaded in many nanoparticles, such as solid lipid carriers and poly (lactide-coglycolide) nanoparticles, it has never been loaded in PUFAs nanoemulsions ${ }^{[13-14]}$. The present study aimed to incorporate ETP into nanoemulsion based on mixing natural oils, Ribes nigrum (black currant) seed oil (BLC) and organic evening primrose oils (EVP) and evaluate its apoptotic and anti-invasion activities on colon cancer cells.

\section{MATERIALS AND METHODS}

\section{Materials:}

ETP was purchased from Ebewe Pharma $\mathrm{GmbH}$ (Unterach, Austria). Organic evening primrose oil (EVP) and BLC were obtained from Chateau Cosmetics Botanical Beauty (Miami, FL, US). The main component of two oils was linoleic acid, gammalinolenic acid, omega 3, 6 and 9. Tween 80 (T80) and span 20 (S20) were acquired from Sigma (Missouri, US). All the tissue culture supplies were purchased from UFC Biotechnology, Inc. (Riyadh, KSA). The 4',6-diamidino-2-phenylindole DAPI dihydrochloride was acquired from Invitrogen (NY, US). Cell Counting Kit- 8 (CCK-8) (Lot. No. LE612) was procured from Dojindo Molecular Technologies, Inc. (JAPAN). Annexin V-FITC Apoptosis Detection Kit (Cat. No. MBS668896) was obtained from MyBioSource, Inc. (San Diego, CA, US). Mitochondria Staining Kit (Cat. No. CS0390) was acquired from Sigma (CA, US).

\section{Synthesis of nanoemulsion formulas:}

The nanoemulsion (BLC-EVP-NANO) was produced by the ultrasonication method. In brief, different volume fractions (v/v) of $2 \%$ BLC, $2 \%$ EVP,7 \% T80, $3 \%$ S20 and $86 \%$ sodium acetate buffer (pH 5) were mixed at $25^{\circ}$. Then, the mixture was sonicated for 20 min until it became clear and transparent with the Omni Sonic Ruptor 4000 Ultrasonic Homogenizer (NY, US). The drug-loaded formulation (ETP-BLCEVP-NANO) was prepared by directly solubilizing the ETP in BLC-EVP-NANO.

\section{Physical characterization of nanoemulsion formulations:}

The absorption spectra for droplet size distributions of BLC-EVP-NANO and ETP-BLC-EVP-NANO were measured at the wavelength (200-600 nm) using Ultraviolet-visible (UV-Vis) spectrophotometer (Thermo Scientific ${ }^{\mathrm{TM}}$, US). The droplet sizes and zeta potentials of the dispersed nanodroplets for BLC-EVPNANO and ETP-BLC-EVP-NANO were assessed using the Zetasizer (Malvern Instruments Ltd., UK).

\section{In vitro drug release:}

The dialysis technique evaluated the in vitro release of the ETP and ETP-BLC-EVP-NANO. Both formulations $(1 \mathrm{ml})$ were transferred into the dialysis bag separately (Cut-off 3.5 Da, Spectra Lab, CA, US). Then, the bag was incubated into the phosphate buffer $(200 \mathrm{ml}, \mathrm{pH}$ 7.4) and stirred at $1 \mathrm{~g}$. Nearly, $1 \mathrm{ml}$ of the buffer was taken at a predetermined interval and replaced by fresh buffer $(1 \mathrm{ml})$. The removed buffer absorbance at a specific time (A1) was measured by using a UVVis spectrophotometer (Thermo Scientific ${ }^{\mathrm{TM}}$, US). The release of ETP (\%) was measured by means of dividing A1 by A0 (absorbance of the initial drug sample added to the dialysis bag) and multiplying by 100 .

\section{Assessment of antitumor activity:}

Cell culture: The colon cancer cell line (HCT116) was purchased from America Type Tissue Culture Collection (Manassas, VA, USA). The $2 \times 10^{6}$ HCT116 cells were plated in a culture flask $\left(25 \mathrm{~cm}^{2}\right)$ containing $5 \mathrm{ml}$ of Dulbecco's modification of eagle's medium (DMEM), complemented with $10 \%$ fetal bovine serum (FBS), $1 \%$ penicillin /streptomycin and incubated at $37^{\circ}$ in a $5 \% \mathrm{CO}_{2}$ humidified atmosphere. For subculture, the DMEM was discarded, cells rinsed with phosphatebuffered saline (PBS) and detached from flasks by adding $1 \mathrm{ml}$ of $0.25 \%$ trypsin. The cells were nourished 
with DMEM until $90 \%$ confluence was obtained.

Cell counting kit-8: The $1 \times 10^{4}$ of HCT116 cells were cultured into 96 -well plate at which each well contained $100 \mu$ of growth medium followed by incubation at $37^{\circ}$ for $24 \mathrm{~h}$ in a $\mathrm{CO}_{2}$ incubator. Then, $200 \mu \mathrm{l}$ of ETP (0.5$3 \mu \mathrm{M})$, BLC-EVP-NANO (0.5- $3 \% \mathrm{v} / \mathrm{v})$ and ETPBLC-EVP-NANO $(0.5-3 \mu \mathrm{M})$ were added to each well and incubated at the same culture conditions. In order to evaluate the cellular proliferation, $5 \mu \mathrm{l}$ of CCK-8 reagent was added in the dark into each well, mixed gently, and incubated for $3 \mathrm{~h}$ at culture conditions. After that, the absorbance (A) of treated cells, media containing cells (control) and media (blank) were assessed in a BioTek Microplate Reader $(\lambda=450 \mathrm{~nm})$. The cellular proliferation (CP \%) was calculated by subsequent calculation:

$\mathrm{CP} \%=((\mathrm{A}$ of treated cells-A of blank $) /(\mathrm{A}$ of control) $) * 100$

Cell morphology characterization: Cells $\left(1 \times 10^{4} /\right.$ well) were planted at $100 \mu \mathrm{l}$ of DMEM into a 96-well. Then, $200 \mu \mathrm{l}$ of the $\mathrm{IC}_{50}$ for ETP, BLC-EVP-NANO, and ETP-BLC-EVP-NANO were added to each well and incubated for $24 \mathrm{~h}$ at $37^{\circ}$. After that, DMEM was removed, cells were rinsed with buffer saline $(100 \mu \mathrm{l})$, fixed by the addition of formaldehyde ( $200 \mu 1,4 \%)$, and stained with $100 \mu \mathrm{l}$ of $5 \%$ Coomassie brilliant blue dye (Fisher Scientific, US) for $10 \mathrm{~min}$. Finally, cells were cleaned with tap water followed by drying overnight at $25^{\circ}$. The morphological alteration of treated cells was detected using a phase-contrast inverted microscope (Olympus, Japan).

Nuclear DNA Staining: Cells ) $5 \times 10^{4} /$ well) were cultured in $500 \mu \mathrm{L}$ of DMEM in a 24 -well plate and incubated for $24 \mathrm{~h}$ at $37{ }^{\circ} \mathrm{C}$. Then, $500 \mu \mathrm{L}$ of the $\mathrm{IC}_{50}$ for each tested formulation was added and incubated for $24 \mathrm{~h}$. Following incubation, cells rinsed with PBS $(300 \mu \mathrm{L})$, fixed via $4 \%$ formaldehyde $(200 \mu \mathrm{L})$, stained for 2 min with DAPI stain $(300 \mu \mathrm{L}, 300 \mathrm{nM})$ and visualized using fluorescent microscope at $437 \mu \mathrm{m}$ (Leica CRT6000, Germany).

Mitochondrial polarization assay: JC1) 5,5',6,6'-tetrachloro-1,1',3,3'-tetraethylbenzimidazolylcarbocyanine iodide) is a cytofluorimetric, lipophilic, cationic dye, used to assed the mitochondrial membrane polarization of HCT116 cells. Briefly, cells ) $1 \times 10^{4} /$ well) were cultured into $100 \mu \mathrm{L}$ of growth medium into 96 well-plates. Then, $200 \mu \mathrm{L}$ of $\mathrm{IC}_{50}$ for various treatments were added to each well. After $24 \mathrm{~h}$ incubation, cells stained by JC-1 solution (JC-1 stain, JC-1buffer solution, and DMEM) and incubated for 30 $\min$ at $37{ }^{\circ} \mathrm{C}$. Finally, the fluorescence was examined at excitation (Ex): $525 \mathrm{~nm} / \mathrm{emission}(\mathrm{Em}): 590$ for red fluorescence (depolarization) and at Ex:490nm/ Em:530 for green fluorescence(hyperpolarization) using fluorescence microplate reader (Synergy ${ }^{\text {TM }}$ HTX, BioTek, US).

Annexin V-FITC/PI assay: HCT116 cells $\left(3 \times 10^{5} /\right.$ well) were cultured at $2 \mathrm{ml}$ of DMEM into six wellplates and incubated at culture conditions. Then, $2 \mathrm{ml}$ of $\mathrm{IC}_{50}$ for each tested formulation was added to each well. Following $24 \mathrm{~h}$ incubation, cells were harvested, rinsed with pre-cold PBS, centrifuged (300 g), re-suspended in $100 \mu \mathrm{l}$ of $1 \mathrm{X}$ binding buffer, and transferred to a flow cytometry tube. Then, $5 \mu \mathrm{l}$ of each FITC and PI added to the cell suspension, incubated for $20 \mathrm{~min}$ and completed with $400 \mu 1$ of binding buffer. Finally, cells evaluated within one hour using flow cytometer (FACS Aria $^{\mathrm{TM}}$ III, BD Biosciences, CA, US).

Nucleosome assays: The ratio of nucleosome was analyzed by utilizing Cell death detection ELISA ${ }^{\text {plus }}$ kit (Lot. No. 19315700) (Mannheim, Germany). Briefly, HCT116 cells ) $1 \times 10^{4} /$ well) were cultivated at $100 \mu \mathrm{L}$ of growth medium in each well of the 96 well-plates and incubated at culture conditions. Then, $200 \mu \mathrm{l}$ of the $\mathrm{IC}_{50}$ of different formulations was added to each well and incubated for $24 \mathrm{~h}$. Following incubation, the plate was spun down (200 g), and the cells were suspended in the lysis solution. The lysates $(20 \mu \mathrm{l})$ were transferred to a streptavidin-coated microplate, complemented with $80 \mu \mathrm{l}$ of immunoreagent, and incubated on a shaker $(10 \mathrm{~g})$ at the dark for $2 \mathrm{~h}$. After that, the ABTS stain was added, incubated on a plate shaker for $10 \mathrm{~min}$ and stopped by ABTS stop solution. The absorbance (A) was determined at $405 \mathrm{~nm}$. The nucleosome ratio was calculated according to the following equation:

Nucleosome ratio $=($ A of the treated cell $($ dead cells $) / A$ of the control cell (viable cells)).

Invasion assay: The $\mathrm{QCM}^{\mathrm{TM}}$ 24-well Collagen-based Invasion Assay (Cat.No.ECM551, Merck KGaA Darmstadt, Germany) was used to evaluate the invasion capability of HCT116 cells. Briefly, the $3 \times 10^{5}$ cells / well were cultured into a 6 -well plate containing $2 \mathrm{ml}$ of growth medium. Then, $2 \mathrm{ml}$ of $\mathrm{IC}_{50}$ for each tested formulation was added to each well. After that, media without FBS $(300 \mu 1)$ was supplemented to each insert and incubated at $25^{\circ}$ for $30 \mathrm{~min}$. Then, $250 \mu \mathrm{l}$ of media were extracted and $250 \mu 1$ of cells suspension was added 
into each insert suspended in $500 \mu 1$ of the media with $10 \% \mathrm{FBS}$ in the lower chamber. Then, the plate was covered and incubated for $24 \mathrm{~h}$ in the $\mathrm{CO}_{2}$ incubator. After that, all non-invaded cells were detached, and the insert was placed in $400 \mu \mathrm{l}$ of cell stain for $20 \mathrm{~min}$. Then, the insert was rinsed, gently cleaned, transferred into $200 \mu \mathrm{l}$ of extraction buffer and left for $15 \mathrm{~min}$ at $25^{\circ}$. Finally, $100 \mu 1$ of the extracted buffer were shifted to a 96-well culture plate for colorimetric measurement at $560 \mathrm{~nm}$. The HCT116 cell invasion was measured according to the subsequent equation:

Relative Invasion $(\%)=(\mathrm{A}$ of treatment/A of control)*100

Statistical analysis: One-way ANOVA was used to estimate the statistical analyses between samples. The $\mathrm{p}$-values were measured according to the pairwise $t$-tests and considered statistically significant when $\mathrm{p}<0.05$. Data were expressed as mean \pm standard deviations for tree determinations $(\mathrm{n}=3)$. The statistical analyses were carried out using MegaStat Excel Software (version 10.3, Butler University, Indianapolis).

\section{RESULTS AND DISCUSSION}

Although the maximum absorptions for each produced formula, BLC-EVP-NANO, and ETP-BLC-EVPNANO, was recorded at the same wavelength $\left(\lambda_{\max }=300 \mathrm{~nm}\right)$, the amounts of absorbances for ETPBLC-EVP-NANO were larger than these of BLCEVP-NANO at each wavelength ( $p<0.001$, fig. 1). The distribution around the maximum absorption for both formulas was implied by measuring the kurtosis which was 2.3 and 1.1 for the absorption curves of BLCEVP-NANO and ETP-BLC-EVP-NANO, respectively which indicate that both absorption spectrums were platykurtic and have a broad distribution of the absorbances at different wavelengths.

The z-average diameters, coefficient of variations and zeta potentials for the formulated nanoemulsions, BLC-EVP-NANO and ETP-BLC-EVP-NANO were summarized in Table 1. The nanodroplet sizes and surface charges of BLC-EVP-NANO were considerably changed when loaded with ETP. According to the measured coefficient of variations among the droplets sizes, the small values for both formulas imply normal and homogeneous distribution among the nanodroplets.

According to fig. 2, $35 \%$ of ETP-BLC-EVP-NANO was released at the initial hour compared to $12 \%$ of the native ETP solution. Following the initial hour, a sustained release of both ETP-BLC-EVP-NANO and native ETP was observed. Within six hours, almost $90 \%$ of the ETP-BLC-EVP-NANO and only $50 \%$ of the ETP were released.

The percentages of the proliferative HCT116 cells subjected to the ETP, BLC-EVP-NANO, and ETP-BLCEVP-NANO at various concentrations $(0.5 \mu \mathrm{M}, 1 \mu \mathrm{M}$, $1.5 \mu \mathrm{M}$ and $3 \mu \mathrm{M}$ ) were set out in fig. 3. Interestingly, the proliferation of BLC-EVP-NANO and ETP-BLCEVP-NANO treated cells were markedly lower than the growth of ETP treated cells at all tested concentrations. The measured half-maximal inhibitory concentration $\left(\mathrm{IC}_{50}\right)$ for BLC-EVP-NANO $(3.1 \pm 1.7) \mu \mathrm{M}$ and ETPBLC-EVP-NANO $(1.7 \pm 1.3) \mu \mathrm{M}$ were strikingly lesser than that of ETP $(18.1 \pm 2.5) \mu \mathrm{M}(\mathrm{p}<0.001)$.

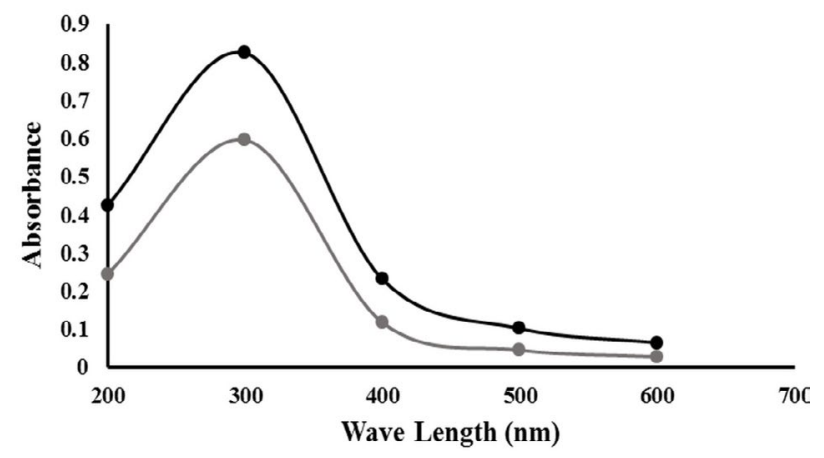

Fig. 1: UV-Vis absorption spectra for particle size distributions of BLC-EVP-NANO and ETP-BLC-EVP-NANO

TABLE 1: THE PHYSICAL CHARACTERISTICS OF THE NANODROPLETS FOR BLC-EVP-NANO AND ETP-BLC-EVP-NANO. THE $\left({ }^{* * *}\right)$ REFERRED TO THE EXTREMELY HIGHLY SIGNIFICANT DIFFERENCE BETWEEN BLC-EVP-NANO AND ETP-BLC-EVPNANO.

\begin{tabular}{lcc}
\hline & BLC-EVP-NANO & ETP-BLC-EVP-NANO \\
\hline $\begin{array}{l}\text { Z-average diameter } \\
(\mathrm{nm})\end{array}$ & $59.42 \pm 2.5$ & $144.6 \pm 5.3^{* * *}$ \\
$\begin{array}{l}\text { Zeta potential }(\mathrm{mV}) \\
\begin{array}{l}\text { Coefficient of } \\
\text { variation }\end{array}\end{array}$ & $-0.183 \pm 0.002$ & $-0.0808 \pm 0.001^{* * *}$ \\
\hline
\end{tabular}

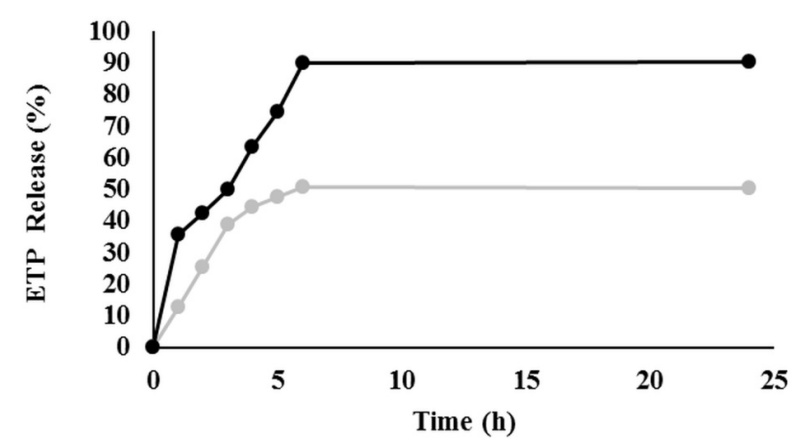

Fig. 2: The release of native ETP and ETP-BLC-EVP-NANO in phosphate buffer (pH 7) at $37^{\circ} \mathrm{C}$ 
As shown in fig. 4 ( $a$ and $b$ ), most of the untreated HCT116 cells (control) displayed a homogenous cell shape, intact cell membrane, and normally rounded nucleus that indicates the cell viabilities. On the other hand, a decrease in cell count, irregular cell shape and membrane blubbing were revealed in cells treated with all formulations. In fact, the signs of late apoptosis, such as nucleus fragmentation and chromatin condensation were higher in cells treated with BLC-EVP-NANO and ETP-BLC-EVP-NANO.

The mitochondrial polarization ratio of HCT116 cells treated with the $\mathrm{IC}_{50}$ of the various formulations was exhibited in fig. 5. The mitochondrial hyperpolarization (high red fluorescence) was detected in the untreated HCT116 cells (control), which was an indication of the cell viability. In contrast, the mitochondrial depolarization, indicated by the lowered red/green fluorescence intensity ratio, was markedly detected in the cells treated with ETP-BLC-EVP-NANO $(p<0.001)$.

The apoptotic stages of the untreated and treated HCT116 cells were categorized according to their ability to stain with annexin V-FITC and PI as shown in the flow cytometry plots (fig. 6). The percentages of the treated HCT116 cells differentiated according to

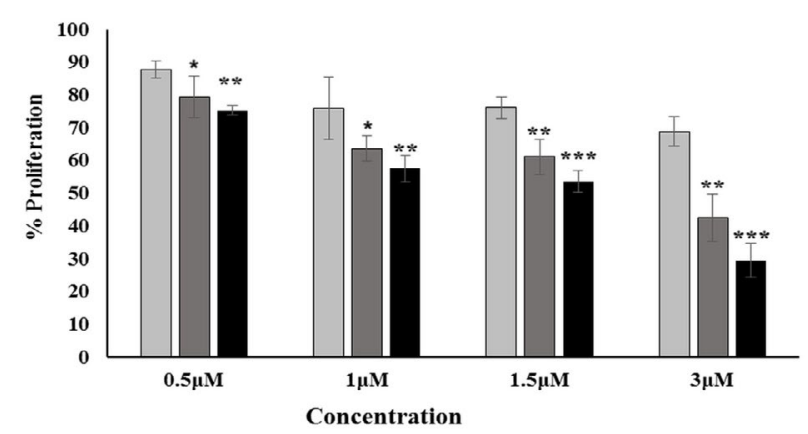

Fig. 3: The effect of different concentrations of ETP, BLC-EVPNANO, and ETP-BLC-EVP-NANO on the HCT116 cellular proliferation. Data were expressed as mean \pm SD $(n=3)$. Error bars represented the \pm SD. One-way ANOVA and independent $t$-test evaluated the significant differences between groups. The ${ }^{*},{ }^{* *}$ and ${ }^{* * * *}$ represented the significant $\left({ }^{*}, p<0.05\right)$, highly significant $(* *, p<0.01)$ and very highly significant $\left({ }^{* * *}, p<\right.$ 0.001) differences between ETP and the other tested formulas

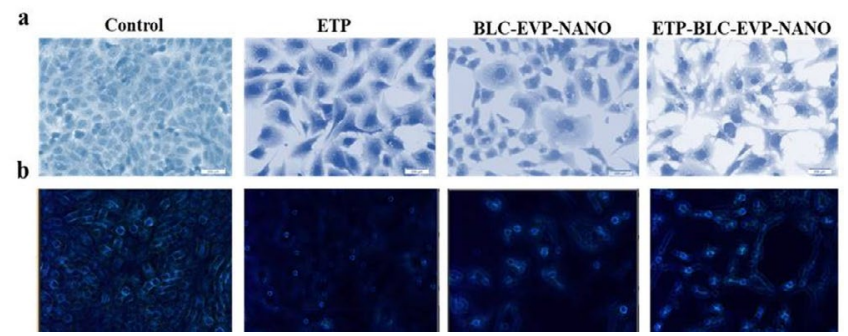

Fig. 4: (a) Light microscopy images of the untreated and treated HCT116 cells stained with Coomassie blue (40X magnification) (b) Fluorescent microscopy images of the untreated and treated HCT116 cells stained with DAPI (20X magnification)

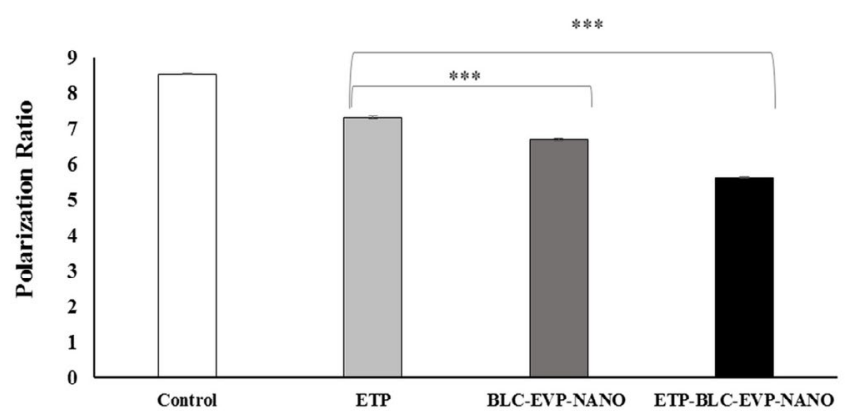

Fig. 5: The polarization ratio (red/green fluorescence intensity) of the control and treated HCT116 cells stained with JC-1. Data were expressed as mean \pm SD $(n=3)$. Error bars displayed the \pm SD. The p-values were assessed by the one-way ANOVA and the pairwise $t$-tests. The $\left({ }^{* * * *}, p<0.001\right)$ represented the very highly significant difference between the variables. All of the treated cells were markedly different from the control $(p<0.001)$

the stages of apoptosis were quantified as illustrated in Table 2. The ETP-BLC-EVP-NANO treatment caused a significant enhancement in the amount of late apoptotic cells and lowered the amount of the viable cells when compared to the ETP and BLC-EVP-NANO treatments.

The ratio of nucleosomes in the cytoplasm of the apoptotic HCT116 cells following treatment with the $\mathrm{IC}_{50}$ of ETP, BLC-EVP-NANO and ETP-BLC-EVPNANO were clarified in fig. 7. The lowest amount of nucleosomes was markedly detected when cells were treated with ETP. In contrast, the ratio of nucleosomes of the cells treated with BLC-EVP-NANO and ETP-BLCEVP-NANO were noticeably increased to $6.8 \pm 0.09$ and $9.7 \pm 0.09$ respectively, when compared to that of ETP treated cells (4.5 \pm 0.06$)$.

The capability of the various formulations to suppress the HCT116 cell invasion was revealed at the microscopy images (fig. 8). The highest inhibition of HCT116 cell invasion was detected with ETP-BLCEVP-NANO treatment $(\mathrm{p}<0.001)$. BLC-EVP-NANO treatment had also considerably impeded the invasion ability of HCT 116 cells when compared to the untreated and ETP treated cells $(\mathrm{p}<0.001)$.

Polyunsaturated fatty acids (PUFAs) as an active agent of natural oils have several properties such as antimicrobial and anti-cancer activity. PUFAs are placed at the front of the biotechnological study owing to the potent activity of these compounds in the pharmacy ${ }^{[9]}$. Using nanoemulsions in cancer therapy has become more promising owing to their larger surface area, superficial charge, bioavailability, specific targeting and easy accumulation in the cancer cell ${ }^{[15]}$. In the current study, both BLC and EVP, which are natural oils enriched with PUFA, were formulated with tween 


\begin{tabular}{lcccc}
\hline & \multicolumn{4}{c}{ Type of cells } \\
\cline { 2 - 5 } & Late apoptotic (Q2) & Early apoptotic (Q4) & Necrotic (Q1) & Viable (Q3) \\
ETP & $37.83 \pm 0.61$ & $36.36 \pm 1.26$ & $5.23 \pm 0.37$ & $20.53 \pm 1.58$ \\
BLC-EVP-NANO & $38.7 \pm 3.91$ & $36.5 \pm 2.59$ & $4.8 \pm 0.61$ & $19.63 \pm 1.51$ \\
ETP-BLC-EVP-NANO & $42.33 \pm 1.72^{* *}$ & $38.5 \pm 1.27$ & $5.66 \pm 1.14$ & $13.46 \pm 0.57^{* *}$ \\
\hline
\end{tabular}

Data were expressed as mean \pm SD $(n=3)$. The $p$-values were assessed using the independent $t$-tests between the variables. The $\left.{ }^{* *}\right)$ referred to the highly significant differences $(0.001 \leq \mathrm{p}<0.01)$ with respect to ETP.

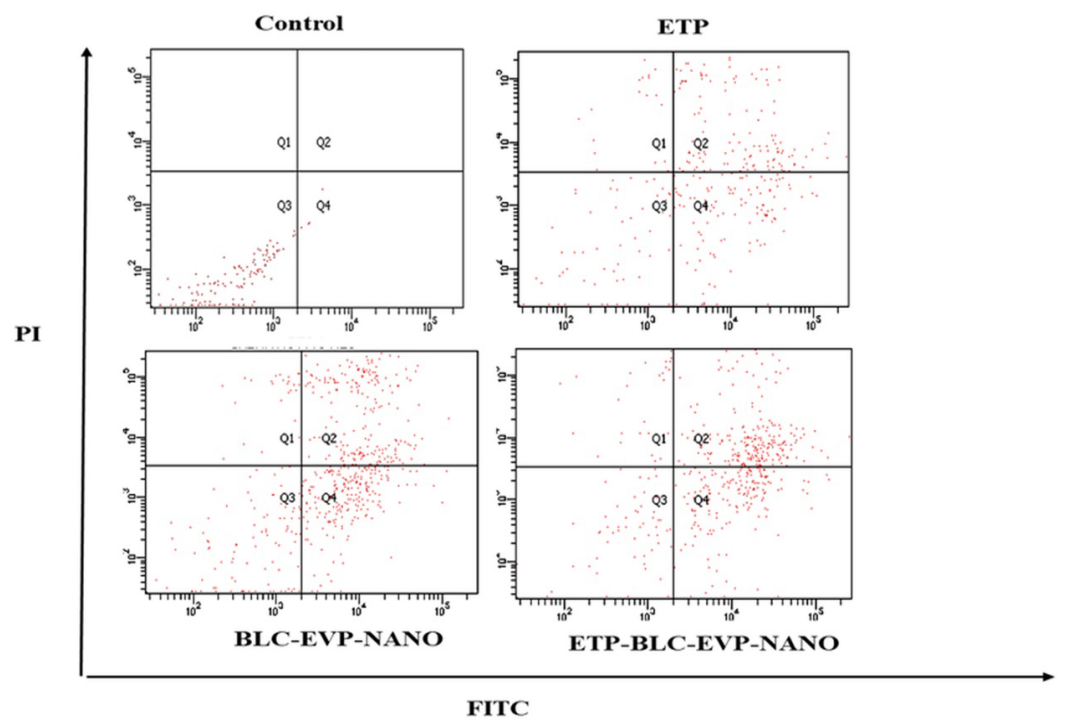

Fig. 6: Annexin V-FITC /PI flow cytometry plots of untreated and treated HCT116 with the IC $_{50}$ of the various formulations

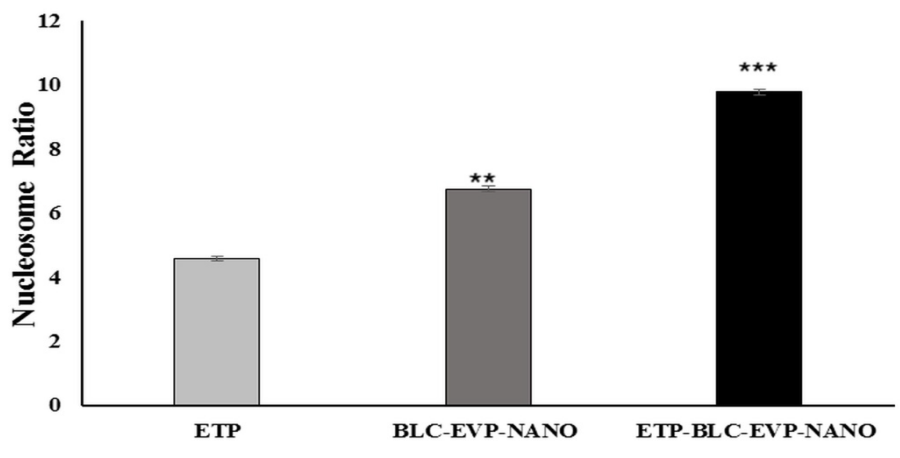

Fig. 7: The ratio of the nucleosome in HCT116 cells subjected to the IC of ETP, BLC-EVP-NANO, and ETP-BLC-EVP-NANO. Error bars represented the \pm SD. The p-values were assessed according to to $^{0}$ the one-way ANOVA and measuring the pairwise $t$-tests. The $\left({ }^{* * *}\right.$ and $\left.^{* *}\right)$ referred to the high $(p<0.01)$ and very highly significant difference $(p<0.001)$ between ETP and the other tested formulas

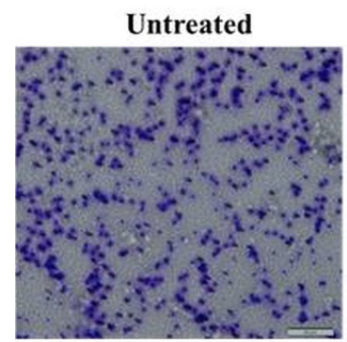

$0 \%$

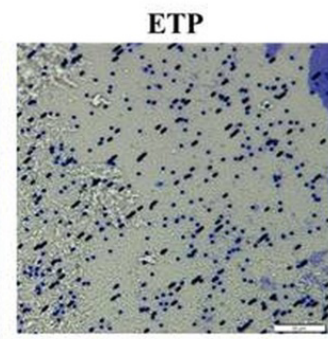

$(35.1 \pm 1.2) \%$
BLC-EVP-NANO

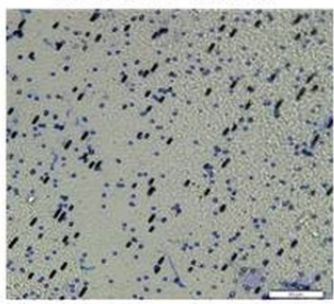

$(49.4 \pm 2.4) \%$
ETP-BLC-EVP-NANO

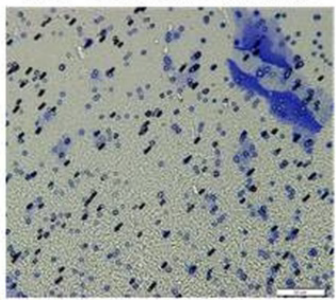

$(54.8 \pm 1.2) \%$

Fig. 8: Light microscopy images of the invasion for the untreated and $24 \mathrm{~h}$-treated HCT116 cells (20X magnification). The percentages of the impeded cells from invasion, subjected to different treatments, are shown below each image 
80 and span 20 in a buffer (pH5) to produce oil-in-water NE with the aim to facilitate the delivery of ETP and improve its antineoplastic effect on the colon cancer cells. The droplet sizes for both produced formulas, BLC-EVP-NANO and ETP-BLC-EVP-NANO, were lower than $150 \mathrm{~nm}$ and distributed in a monodisperse pattern. The noticeable increase of droplet size for ETPBLC-EVP-NANO relative to BLC-EVP-NANO might be evidence that ETP was trapped inside the BLCEVP-NANO formula. In agreement with our result, a previous study revealed that the incorporated ifosfamide with camphor-NE displayed a larger droplet size than the blank camphor-NE ${ }^{[16]}$. Likewise, the UV-Vis measurement showed that the absorptions of the BLCEVP-NANO were obviously increased when loaded with ETP. The previous study on gold nanoparticles carried out by Doak et al. ${ }^{[17]}$ exhibited that the larger particle size was combined with larger absorbance at the long wavelengths. It is worth pointing out that the small and monodisperse size distribution of ETP-BLCEVP-NANO might be leading to the complete release of ETP-BLC-EVP-NANO subsequently $24 \mathrm{~h}$.

Regarding HCT116 cell proliferation, the $\mathrm{IC}_{50}$ of ETPBLC-EVP-NANO (1.7 \pm 1.3$)$ was markedly decreased with around ten-folds than the ETP $(18.1 \pm 2.5) \mu \mathrm{M}$. Narrowest droplet size with a monodisperse distribution of ETP-BLC-EVP-NANO was expected to raise the cellular uptake of the formulation and enhanced its adhesion to the cancer cells ${ }^{[18]}$. Additionally, the decline in the proliferating of HCT116 cells with ETP-BLCEVP-NANO treatment could be accredited to the synergistic effect of the natural active agents of oils and ETP. Similarly, our recent study conducted on lung cancer cell line A549 cells showed a decrease in cellular growth when cells treated with etoposide encapsulated nanoemulsion prepared in sodium phosphate buffer $(\mathrm{pH}$ 7) compared to the free ETP which has no noteworthy effect on A549 cells ${ }^{[19]}$. The anti-inflammatory and antiproliferative effect is one of the most pharmacological effects of EVP oil ${ }^{[20]}$.

The early event of apoptosis in HCT116 cells treated with ETP, BLC-EVP-NANO and ETP-BLC-EVPNANO was detected in the morphological changes observations using light microscopy. The changes, such as irregular cell shape and membrane blubbing were higher in the treated HCT116 cells. Besides, the mitochondrial depolarization, which was evaluated by lowering the red/green fluorescence intensity ratio was higher with ETP-BLC-EVP-NANO treatments.
Along with the mitochondria, the nucleus was the most critical organelles for cell proliferation and apoptosis. Therefore, the main goal of cancer therapy is to target the nucleus that controlled the processes leading to cell apoptosis $^{[21]}$. Interestingly, the increased percentage of late apoptotic cells with ETP-BLC-EVP-NANO treatments could be an indication that the ETP-BLCEVP-NANO formula was affecting the DNA. This finding was confirmed with the cell death assay, which showed the maximum amounts of the nucleosomes at the cytoplasm of apoptotic HCT116 cells treated with the ETP-BLC-EVP-NANO. Consequently, loading ETP on natural oil-based nanoemulsions possibly will enhance the induction of apoptosis in cancer cells. Recently, several studies have reported that incorporating chemotherapeutic agents into nanoemulsions based on carrot seed oil, garlic oil, ginger oil and flaxseed oil had potentiated the effectiveness of the chemotherapeutic agent toward cancer cells ${ }^{[22-23-24-25]}$. The invasion is a critical component in the escape and propagation of tumor cells from the main site into distant organs ${ }^{[26]}$. In the present study, treatment with ETP-BLC-EVPNANO and BLC-EVP-NANO had reduced the HCT116 invasion, when compared to the untreated and ETP treated cells which indicate the major role of BLC and EVP included in the NE formula. The EVP is a valuable source of linoleic acid and gamma-linolenic acid. The gamma-linolenic acid causes an increase in the expression of the nm-23 metastasis-suppressor gene in cancer cells, which promotes the inhibition of angiogenesis, cancer cell migration, and subsequently, cancer metastasis ${ }^{[27,20]}$.

In conclusion, a novel nanoemulsion formula BLCEVP-NANO was designed by combining natural oils, Ribes nigrum (black currant) seed oil (BLC), and organic evening primrose oil (EVP) with an emulsifying agent. Incorporating ETP into a new formula had augmented its anti-proliferation effect on HCT116 colon cancer cells. The combination of ETP with the natural oils in ETP-BLC-EVP-NANO had enhanced the induction of apoptosis and the inhibition of the invasion capability among colon cancer cells. According to our findings, molecular mechanisms of the anticancer activity of the BLC-EVP-NANO and ETP-BLC-EVP-NANO formulas need to be studied and further investigations in animal models are also required.

\section{Conflict of Interest:}

The authors declare that they have no conflict of interest. 


\section{REFERENCES}

1. Bray F, Ferlay J, Soerjomataram I, Siegel RL, Torre LA, Jemal A. Global cancer statistics 2018: GLOBOCAN estimates of incidence and mortality worldwide for 36 cancers in 185 countries. CA Cancer J Clin 2018;68(6): 394-424.

2. Favoriti P, Carbone G, Greco, Pirozzi F, Pirozzi REM, Corcione F. Worldwide burden of colorectal cancer: a review. Updates Surg 2016;68(1):7-11.

3. Razak S, Afsar T, Almajwal A, Alam I, Jahan S. Growth inhibition and apoptosis in colorectal cancer cells induced by Vitamin D-Nanoemulsion (NVD): involvement of Wnt/ $\beta$ catenin and other signal transduction pathways. Cell Biosci 2019;9(1):15.

4. Yang $\mathrm{X}, \mathrm{Xu} \mathrm{Y}$, Wang $\mathrm{T}$, Shu D, Guo P, Miskimins $\mathrm{K}$, et al. Inhibition of cancer migration and invasion by knocking down delta-5-desaturase in COX-2 overexpressed cancer cells. Redox Biol 2017;11:653-62.

5. Zou H, Li L, Carcedo IG, Xu ZP, Monteiro M, Gu W. Synergistic inhibition of colon cancer cell growth with nanoemulsionloaded paclitaxel and PI3K/mTOR dual inhibitor BEZ235 through apoptosis. Int J Nanomed 2016;11:1947.

6. Reddy LH, Adhikari JS, Dwarakanath BSR, Sharma RK, Murthy RR. Tumoricidal effects of etoposide incorporated into solid lipid nanoparticles after intraperitoneal administration in Dalton's lymphoma bearing mice. AAPS J 2006;8(2):E254-62.

7. Praveen Kumar G, Divya A. Nanoemulsion based targeting in cancer therapeutics. Med Chem 2015;5:272-84.

8. Desbois A, Lawlor K. Antibacterial activity of long-chain polyunsaturated fatty acids against Propionibacterium acnes and Staphylococcus aureus. Mar Drugs. 2013;11(11):4544-57.

9. Sayegh F, Elazzazy A, Bellou S, Moustogianni A, Elkady A I, Baeshen MN, et al. Production of polyunsaturated single cell oils possessing antimicrobial and anticancer properties. Ann Microbiol 2016; 66(3):937-48.

10. Xu Y, Qian SY. Anti-cancer activities of $\omega-6$ polyunsaturated fatty acids. Biomed J 2014;37(3):112.

11. Jaiswal M, Dudhe R, Sharma P. Nanoemulsion: an advanced mode of drug delivery system. Biotech 2015;5(2):123-7.

12. Lovelyn C, Attama AA. Current state of nanoemulsions in drug delivery. J Biomater Nanobiotechnol 2011;2(5):626.

13. Mitra M, Dilnawaz F, Misra R, Harilal A, Verma R S, Sahoo S K, et al. Toxicogenomics of nanoparticulate delivery of etoposide: potential impact on nanotechnology in retinoblastoma therapy. Cancer Nanotechnol 2011;2(1):21

14. Zhang S, Lu C, Zhang X, Li J, Jiang H. Targeted delivery of etoposide to cancer cells by folate-modified nanostructured lipid drug delivery system. Drug Deliv 2016;23(5):1838-45.

15. Sánchez-López E, Guerra M, Dias-Ferreira J, LopezMachado A, Ettcheto M, Cano A, et al. Current Applications of Nanoemulsions in Cancer Therapeutics. J Nanomater 2019;9(6):821.

16. AlMotwaa SM, Alkhatib MH, Alkreathy HM. Nanoemulsionbased camphor oil carrying ifosfamide: preparation, characterization, and in-vitro evaluation in cancer cells. Int $\mathrm{J}$ Pharm Sci Res 2019;10(4):2018-26.

17. Doak J, Gupta R, Manivannan K, Ghosh K, Kahol P. Effect of particle size distributions on absorbance spectra of gold nanoparticles. Physica E Low Dimens Syst Nanostruct 2010;42(5):1605-9.

18. Alkhatib MH, AlMotwaa SM, Alkreathy HM. Incorporation of ifosfamide into various essential oils-based nanoemulsions ameliorates its apoptotic effect in the cancers cells. Sci Rep 2019;9(1):695.

19. Al-Hashemi SM, Alkhatib MH, Gashlan HM. Incorporating Etoposide into PUFA-rich Oils Nanoemulsion Potentiates its Inhibitory Effect on the Cellular Growth of A549 NonSmall Cell Lung Cancer Cells, Biosci Biotech Res Comm 2020;13(1):106-112.

20. Timoszuk M, Bielawska K, Skrzydlewska E. Evening Primrose (Oenothera biennis) Biological Activity Dependent on Chemical Composition. Antioxidants 2018;7(8):108.

21. Dam DHM, Lee JH, Sisco PN, Co DT, Zhang M, Wasielewski $\mathrm{MR}$, et al. Direct observation of nanoparticle-cancer cell nucleus interactions. ACS Nano 2012;6(4):3318-26.

22. Al-Otaibi WA, Alkhatib MH, Wali AN. Cytotoxicity and apoptosis enhancement in breast and cervical cancer cells upon coadministration of mitomycin $\mathrm{C}$ and essential oils in nanoemulsion formulations. Biomed Pharmacother 2018;106:946-955.

23. Alkhatib MH, Al-Otaibi WA, Wali AN. Antineoplastic activity of mitomycin $\mathrm{C}$ formulated in nanoemulsions-based essential oils on HeLa cervical cancer cells. Chem Biol Interact 2018;291:72-80.

24. Alkhatib MH, Alnahdi NS, Backer WS. Antitumor activity, hematoxicity and hepatotoxicity of sorafenib formulated in a nanoemulsion based on the carrot seed oil. Int J Life Sci Pharma Res 2018;8:50-7.

25. Alkhatib MH, Nori DA, Al-Ghamdi MA. Antitumor Activity and Hepatotoxicity effect of sorafenib incorporated into nanoemulsion formulated with flaxseed oil. Int J Pharm Res Allied Sci 2017;6:175-88.

26. Asif M, Yehya A H S, Al-Mansoub MA, Revadigar V, Ezzat $\mathrm{MO}$, Ahamed MBK, et al. Anticancer attributes of Illicium verum essential oils against colon cancer. S Afr J Bot 2016;103:156-61.

27. Marshall JC, Lee JH, Steeg PS. Clinical-translational strategies for the elevation of Nm23-H1 metastasis suppressor gene expression. Mol Cell Biochem 2009;329(1):115-20. 